\title{
Synthesis and Physicochemical Properties of Two-Dimensional Gallium Sulfide Crystals
}

Chuanfang John Zhang ${ }^{1,2^{\star}}$, Xinhua Liu ${ }^{4}$ and Valeria Nicolosi ${ }^{1,2,3^{*}}$

1 Centre for Research on Adaptive Nanostructures and Nanodevices (Crann) \& Advanced Materials Bio-Engineering Research Centre (Amber), Trinity College Dublin, Dublin 2, Ireland

2 School of Chemistry, Trinity College Dublin, Dublin 2, Ireland

3 School of Physics, Trinity College Dublin, Dublin 2, Ireland

${ }^{4}$ Department of Chemistry, Tongji University, Shanghai, China

"Corresponding authors: Zhang CJ, Centre for Research on Adaptive Nanostructures and Nanodevices (Crann) \& Advanced Materials Bio-Engineering Research Centre (Amber), Trinity College Dublin, Dublin 2, Ireland, Tel: +353-(0)1-896-4837; E-mail: zhangjc@tcd.ie

Nicolosi V, Centre for Research on Adaptive Nanostructures and Nanodevices (Crann) \& Advanced Materials Bio-Engineering Research Centre (Amber), Trinity College Dublin, Dublin 2, Ireland, Tel: + 353 (0)1 896 4480; E-mail: nicolov@tcd.ie

Rec date: April 04, 2016, Acc date: April 29, 2016, Pub date: May 2, 2016

Copyright: @ 2016 Zhang CJ, et al. This is an open-access article distributed under the terms of the Creative Commons Attribution License, which permits unrestricted use, distribution, and reproduction in any medium, provided the original author and source are credited.

\begin{abstract}
Two-dimensional (2D) materials possess exotic properties and have been received huge research interests. The group III-VI semiconducting nanosheets (NS) in particular, have been viewed as promising candidates for ultrathin and flexible optoelectronic devices and also for energy storage device. Liquid-phase exfoliation (LPE) could scalably synthesize 2D NS with good quality but in a much lower cost. This mini-review has briefly summarized the recent synthesis and application of 2D NS, with focuses on the LPE approach and fabrication of 2D NS/carbon conductive composite. The future electrochemical studies of 2D NS/carbon composite for Li-ion batteries anode has also been proposed.
\end{abstract}

Keywords Two-dimensional; Nanosheet; Liquid-phase exfoliation; Lithium-ion battery, Composite

\section{Introduction}

Recently, two-dimensional (2D) materials have attracted huge research attention due to their exotic properties [1-4]. 2D materials, such as MoS2, [5,6] WSe2, [7] stack as thin layered crystals, intriguing huge research ranging from sensing [8,9] to energy applications [10]. In addition to them, the group III-VI semiconducting nanosheets (NS) in particular, have been viewed as promising candidates for ultrathin and flexible optoelectronic devices and also for energy storage device [11-13]. For example, layered metal chalcogenides ( $\mathrm{MC}, \mathrm{M}=\mathrm{Ga}$, In, Ta; $\mathrm{C}=\mathrm{S}$, Se, Te) NS exhibit remarkable mechanical, thermal, anisotropic optical and electronic advantages [14-16].

Pioneering work on the synthesis and properties of these MC NS have been reported over the past few years $[17,18]$. Late et al. [19] reported the $2 \mathrm{D}$ single-sheet $\mathrm{GaS}$ and $\mathrm{GaSe}$ transistors via a micromechanical cleavage technique, demonstrating excellent electron differential mobility. Hu et al. [20] obtained ultrathin GaSe NS by mechanical cleavage-solvent exfoliation method, which demonstrated fast response, high responsivity and quantum efficiency in photodetectors. The layered GaSe NS can also be used as a Li-ion battery (LiB) anode [21]. Liu et al. [22] reported multilayer GaTe flakes which exhibit a higher photoresponsivity than graphene, MoS2, and other layered compounds, showing promising applications in optoelectronic and photosensitive devices. 2D InSe ultrathin sheets have been reported as a promising candidate in optoelectronic devices, such as field-effect transistors and highly responsive photodetectors [23]. Tamalampudi et al. [18] fabricated few-layered InSe on rigid
( $\mathrm{SiO} 2 / \mathrm{Si}$ plate) and flexible (polyethylene terephthalate) substrates, yielding superior photoresponsivities of conducting broad band photodetection from 450 to $785 \mathrm{~nm}$.

It's worth mentioning that any of these applications requires large amount of MC NS, rendering the necessity of scalable production of MC NS. Typically, the layered semiconducting crystals are held together by weak van der Waals forces. One could overcome this force by applying the sonic energy to the crystals, either via bath or tip sonication, to obtain MC NS in large quantities with few-layer enriched dispersion. Such a process also termed as "liquid phase exfoliation", which possesses clear advantages over the traditional mechanical/chemical exfoliation approaches $[16,24]$. Moreover, the NS size could be selected by controlled centrifugation of the exfoliated dispersion, as shown in Figure 1. This is important, as the size of NS strongly determines the optoelectronic properties [25]. 
Page 2 of 3

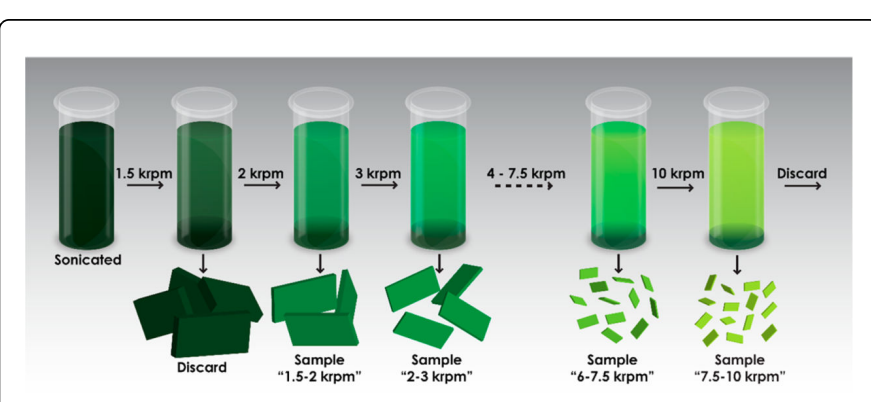

Figure 1: Size selection mechanism using controlled centrifugation approach. Reproduced with permission from ref. 10. Copyright 2016, American Chemical Society.

As a representative member of the layered MC family, gallium sulfide, GaS, shows promising applications in photoelectronics and gas sensing [26,27]. The production of GaS NS is, however, limited by quantities and NS stability [28]. As indicated previously, physical exfoliation of layered $\mathrm{GaS}$ by micromechanical cleavage leads to low throughput [28]. On the other hand, chemical exfoliation would result in a quick NS degradation [29]. In 2015, Coleman et al. [24] obtained GaS NS by exfoliating the commercial crystals in a range of solvents, with GaS-NMP being the optimized system. The exfoliated GaS NS were further processed into electrodes for electrocatalysis application, whose performances strongly depend on the size of NS, as shown in Figure 2 [30].

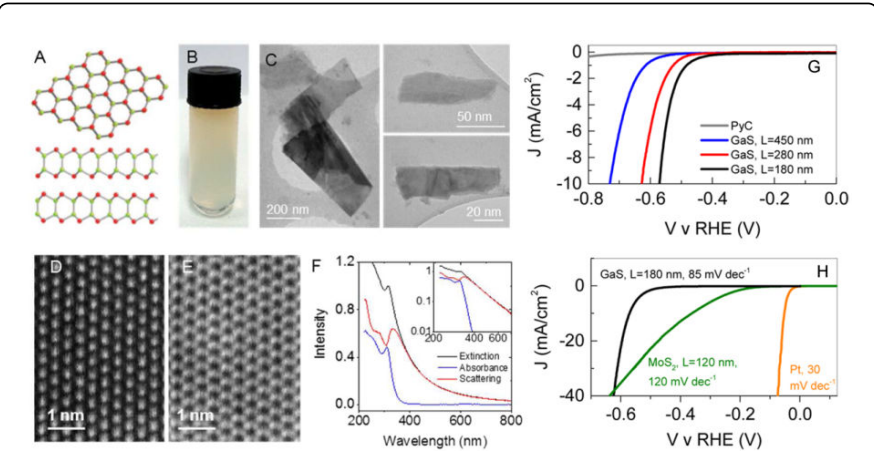

Figure 2: GaS NS synthesized by liquid-phase exfoliation method and its application in hydrogen evolution. Reproduced with permission from ref. 17. Copyright 2015, American Chemical Society.

On the other hand, the multi-valance of $\mathrm{Ga}$ and $\mathrm{S}$ enables multiple charge-transfer reactions and high theoretical capacity as a LiB anode. The semiconducting nature of $\mathrm{GaS}$ could be compensated by adding conductive agents, such as carbon onions, carbon nanotubes, graphene, etc. Ding Q et al. [29] deposited GaSx thin films onto singlewalled carbon nanotubes (SWCNT) using an atomic depositon method. The discharge capacity of the composite reached $575 \mathrm{mAh} \mathrm{g-1}$ per electrode and $770 \mathrm{mAh}$ g-1 per GaSx at $120 \mathrm{~mA}$ g-1. On the other hand, the GaS/conductive composite could explore the high theoretical capacity of $\mathrm{GaS}$, fast electron transport paths of the conductive network and high ionic conductivity in the 2D NS and porous nanostructure, possibly rendering the composite with high capacities and excellent rate capabilities.
We also note that, it is important to clarify the electrochemical reactions by gaining a more thoroughful understanding during charge/ discharge process. For example, by conducting in-situ X-ray diffraction (XRD) we are able to observe the formation of new phases and their reversibility during charge/discharge, and corelate to the electrochemical kinetics. High-resolution transmission electron microscopy (TEM) would also be necessary for directly observation of new crystals post cycling. In doing so, we are able to define the sources of the high capacity, either from $\mathrm{Li}+$ intercalation, or from conversion, or alloying process.

Clearly, the GaS NS/conductive composite represents only one combination of $\mathrm{Ga}, \mathrm{S}$ and conductive agent. Actually, various combinations, such as GaSe, GaTe, InSe NS based composite could be easily fabricated. The compositions, electrode thickness and NS size of the electrode could be easily controlled to maximize the specific capacities and optoelectronic properties. Such an exciting materials configuration and nanostructure certainly deserves further investigation.

\section{References}

1. Chhowalla M, Shin HS, Eda G, Li LJ, Loh KP, et al. (2013) The chemistry of two-dimensional layered transition metal dichalcogenide nanosheets. Nat Chem 5: 263-275.

2. M.Q. Zhao, Q. Zhang, J.Q. Huang, F. Wei (2012) Hierarchical nanocomposites derived from nanocarbons and layered double hydroxides - properties, synthesis, and applications. Adv Funct Mater 2: 675-694.

3. M.B. Erande, S.R. Suryawanshi, M.A. More, D.J. Late (2015) Electrochemically exfoliated black phosphorus nanosheets - prospective field emitters. Eur J Inorg Chem 19: 3102-3107.

4. D. Chakravarty, D.J. Late (2015) Exfoliation of bulk inorganic layered materials into nanosheets by the rapid quenching Method and their electrochemical performance. Eur J Inorg Chem 1: 1973-1980.

5. Late DJ, Shaikh PA, Khare R, Kashid RV, Chaudhary M, et al. (2014) Pulsed laser-deposited MoSâ,, thin films on W and Si: field emission and photoresponse studies. ACS Appl Mater Interfaces 6: 15881-15888.

6. Late DJ, Liu B, Matte HS, Dravid VP, Rao CN (2012) Hysteresis in singlelayer MoS2 field effect transistors. ACS Nano 6: 5635-5641.

7. D. Chakravarty, D.J. Late (2015) Microwave and hydrothermal syntheses of WSe 2 micro/nanorods and their application in supercapacitors. RSC Advances 5: 21700-21709.

8. Kannan PK, Late DJ, Morgan H, Rout CS (2015) Recent developments in $2 \mathrm{D}$ layered inorganic nanomaterials for sensing. Nanoscale 7: 13293-13312.

9. S.R. Kadam, D.J. Late, R.P. Panmand, M.V. Kulkarni, L.K. Nikam, et al. (2015) Nanostructured 2D MoS2 honeycomb and hierarchical 3D CdMoS4 marigold nanoflowers for hydrogen production under solar light. J Mater Chem A 3: 21233-21243.

10. D.J. Late, C.S. Rout, D. Chakravarty, S. Ratha, (2015) Emerging energy applications of two-dimensional layered materials. Can Chem Trans 3: 118-157.

11. Gong Y, Lin J, Wang X, Shi G, Lei S, et al. (2014) Vertical and in-plane heterostructures from WS2/MoS2 monolayers. Nat Mater 13: 1135-1142.

12. D.J. Late, B. Liu, H. Matte, C. Rao, V.P. Dravid, (2012) Emerging energy applications of two-dimensional layered materials. Adv Funct Mater 2: 1894-1905.

13. U.S. Shenoy, U. Gupta, D.S. Narang, D.J. Late, U. V. Waghmare, et al. (2016) Electronic structure and properties of layered gallium telluride. Chem Phys Lett 65: 148-154.

14. V. Brudnyi, S.Y. Sarkisov, A. Kosobutsky (2015) Electronic properties of GaSe, InSe, GaS and GaTe layered semiconductors: charge neutrality level and interface barrier heights. Semicond Sci Technol 30: 115019. 
Citation: Zhang CJ, Liu X, Nicolosi V (2016) Synthesis and Physicochemical Properties of Two-Dimensional Gallium Sulfide Crystals.

Page 3 of 3

15. D. Chakravarty, P. Kumar, V.S. Ugale, D.J. Late (2015) Microwave-assisted synthesis of few-layered TaTe2 and its application as supercapacitor. Eur J Inorg Chem 9: 1598-1603.

16. D.J. Late (2016) Liquid exfoliation of black phosphorus nanosheets and its application as humidity sensor. Microporous Mesoporous Mater 225 494-503.

17. Zhou Y, Nie Y, Liu Y, Yan K, Hong J, et al. (2014) Epitaxy and photoresponse of two-dimensional GaSe crystals on flexible transparent mica sheets. ACS Nano 8: 1485-1490.

18. Tamalampudi SR, Lu YY, Kumar UR, Sankar R, Liao CD, et al. (2014) High performance and bendable few-layered InSe photodetectors with broad spectral response. Nano Lett 14: 2800-2806.

19. Late DJ, Liu B, Luo J, Yan A, Matte HS, et al. (2012) GaS and GaSe ultrathin layer transistors. Adv Mater 24: 3549-3554.

20. Hu P, Wen Z, Wang L, Tan P, Xiao K (2012) Synthesis of few-layer GaSe nanosheets for high performance photodetectors. ACS Nano 6: 5988-5994.

21. Jeong JH, Jung DW, Oh ES (2014) Lithium storage characteristics of a new promising gallium selenide anodic material. J Alloys Comp 61: 42-45.

22. Liu F, Shimotani H, Shang H, Kanagasekaran T, Zólyomi V, et al. (2014) High-sensitivity photodetectors based on multilayer GaTe flakes. ACS Nano 8: 752-760.

23. J. Lauth, F.E. Gorris, M. Samadi Khoshkhoo, T. Chassé, W. Friedrich, et al (2016) Solution-processed two-dimensional ultrathin InSe nanosheets. ACS chemmater 28: 1728-1736.
24. Coleman JN, Lotya M, O'Neill A, Bergin SD, King PJ, et al. (2011) Twodimensional nanosheets produced by liquid exfoliation of layered materials. Science 331: 568-571.

25. Backes C, Szyd A, Yuan S, Owska BM, Harvey A, et al. (2016) Production of highly monolayer enriched dispersions of liquid-exfoliated nanosheets by liquid cascade centrifugation. ACS Nano 10: 1589-1601.

26. Hu P, Wang L, Yoon M, Zhang J, Feng W, et al. (2013) Highly responsive ultrathin $\mathrm{GaS}$ nanosheet photodetectors on rigid and flexible substrates. Nano Lett 13: 1649-1654.

27. Yang S, Li Y, Wang X, Huo N, Xia JB, et al. (2014) High performance fewlayer $\mathrm{GaS}$ photodetector and its unique photo-response in different gas environments. Nanoscale 6: 2582-2587.

28. Kipperman, G. Van der Leeden (1968) Photo-conductivity and photo Hall-effect measurements on gallium sulphide single crystals. Solid State Commun 6: 657-662.

29. Ding Q, Meng F, English CR, Cabán-Acevedo M, Shearer MJ, et al. (2014) Efficient photoelectrochemical hydrogen generation using heterostructures of Si and chemically exfoliated metallic MoS2. J Am Chem Soc 136: 8504-8507.

30. Harvey, C. Backes, Z. Gholamvand, D. Hanlon, D. McAteer, et al. (2015) Mater preparation of gallium sulfide nanosheets by liquid exfoliation and their application as hydrogen evolution catalysts. Chem Mater 27: 3483-3493. 